\title{
Modelling Acute Renal Failure using Blood and Breath Biomarkers in Rats
}

Katherine T. Moorhead ${ }^{1}$, Jonathan V. Hill ${ }^{2}$, J. Geoffrey Chase ${ }^{3}$, Christopher E. Hann $^{3}$, Jennifer M. Scotter ${ }^{4}$, Malina K. Storer ${ }^{4}$, Zoltan H. Endre ${ }^{2}$

${ }^{1}$ Corresponding Author: Department of Mechanical Engineering, University of Canterbury, Private Bag 4800, Christchurch 8140, New Zealand

(Tel: 0064-3-364-2987; e-mail: ktm19@student.canterbury.ac.nz)

${ }^{2}$ Department of Medicine, University of Otago Christchurch School of Medicine, P.O. Box 4345, Christchurch 8140, New Zealand

${ }^{3}$ Department of Mechanical Engineering, University of Canterbury, Private Bag 4800, Christchurch 8140, New Zealand

${ }^{4}$ Syft Technologies Ltd., P.O. Box 28149, Christchurch 8242, New Zealand

Keywords: Renal Function, Breath Biomarkers, Integral Fitting Method, Model-based Approximation, Tracer Kinetics, Differential Equations

This research was conducted on a commercially available SIFT-MS instrument provided by Syft Technologies Ltd., and was supported by a New Zealand Tertiary Education Commission Top Achiever Doctoral Scholarship (UOCX5011).

This study was approved was by the New Zealand Animal Ethics Committee (C09/07601). 


\section{Abstract}

This paper compares three methods for estimating renal function, as tested in rats. Acute Renal Failure (ARF) was induced via a 60-minute bilateral renal artery clamp in 8 Sprague-Dawley rats and renal function was monitored for 1 week post-surgery. A two-compartment model was developed for estimating glomerular filtration via a bolus injection of a radio-labelled inulin tracer, and was compared with an estimated creatinine clearance method, modified using the Cockcroft-Gault equation for rats. These two methods were compared with Selected Ion Flow Tube-Mass Spectrometry (SIFT-MS) monitoring of breath analytes. Determination of renal function via SIFTMS is desirable since results are available non-invasively and in real time. Relative decreases in renal function show very good correlation between all 3 methods $\left(\mathrm{R}^{2}=0.84,0.91\right.$ and 0.72 for breath-inulin, inulin-creatinine, and breath-creatinine correlations, respectively), and indicate good promise for fast, non-invasive determination of renal function via breath testing. 


\section{Introduction}

Current clinical measurements of renal function rely on at least daily measurements of plasma creatinine. However, in Acute Renal Failure (ARF), plasma creatinine levels can be very slow to react to changes in GFR, which can result in delays of 1-3 days in the diagnosis of renal failure in clinical situations [1, 2], and thus lead to unacceptable delays in instituting treatment. It is these delays which have lead to the American Association of Nephrologists to call for a concerted effort at discovery of new and earlier biomarkers of ARF. Consequently, there have been no improvements in mortality in the management of ARF for over 50 years, despite the availability of many useful experimentally effective treatments [3].

Selected Ion Flow Tube-Mass Spectrometry (SIFT-MS) is an analytical technique for the real-time quantification of Volatile Organic Compounds (VOCs) in air or breath samples. By analyzing changes in VOC concentrations in breath over time, SIFT-MS can offer fast diagnosis of various conditions and diseases non-invasively and in realtime. Hence, if renal failure could be assessed at its onset via breath biomarkers, this technology would enable regular, easy assessment and monitoring.

In this study, ARF was induced in 8 Sprague-Dawley rats. Renal function was monitored for 1 week following induction of ARF via plasma creatinine measurements, SIFT-MS breath sampling, and radio-labelled inulin clearance tests. The goal was to correlate changes in breath biomarkers with the current clinical and model-based gold standards. From these results, the potential for SIFT-MS technology was assessed. 


\subsection{Glomerular Filtration Rate (GFR)}

Glomerular Filtration Rate refers to the volume of plasma cleared by filtration through the glomerular capillaries into Bowman's space of the kidney per unit time (Fig. 1), and is generally accepted as the best overall estimation of kidney function. Any substance that is freely filtered by the glomerulus and is not secreted or reabsorbed by the kidneys can be used to measure GFR.

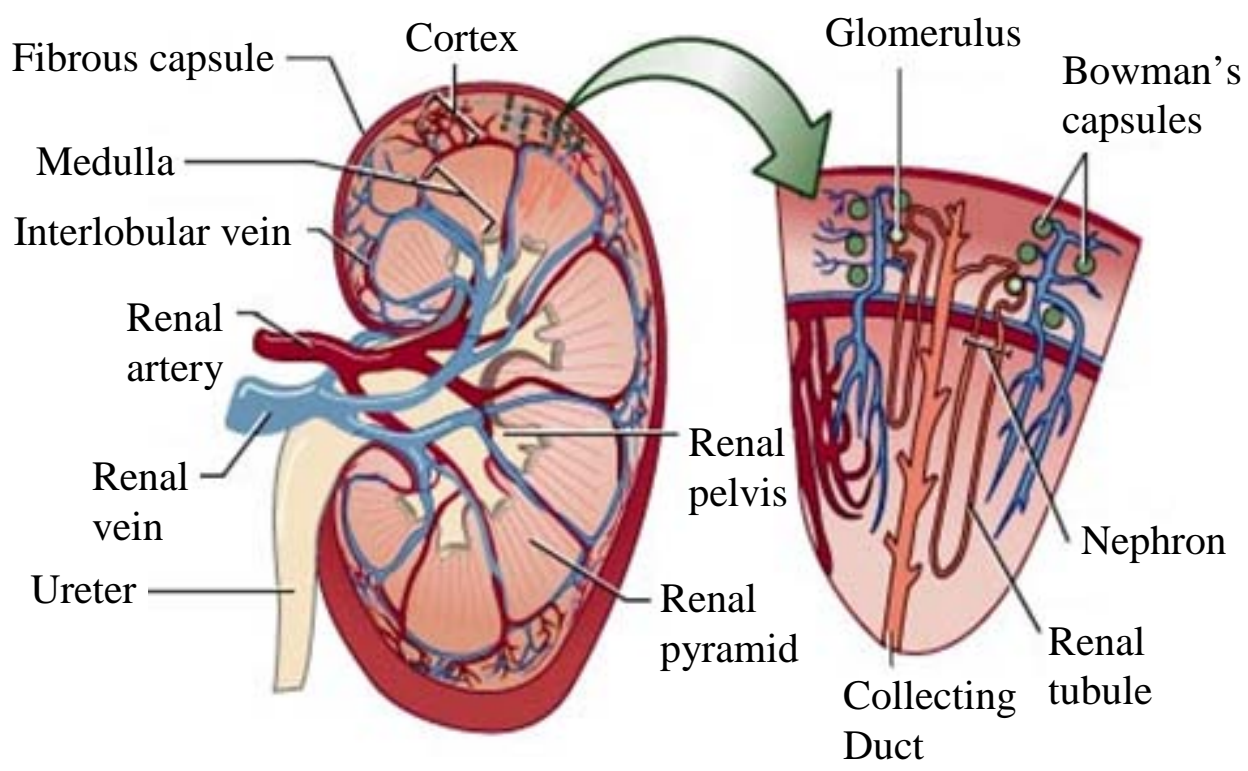

Fig. 1. Kidney Anatomy [4]

\subsection{Exogenous Markers for measuring GFR}

If an exogenous filtration marker, such as inulin, is used to measure GFR, it is injected into the plasma, and its clearance through the kidney can be measured using either constant infusion or bolus techniques [5-8]. Using the constant infusion technique, the exogenous marker is injected into the plasma at a constant rate until a steady state is achieved. At this point, Equation (1) is applied, where plasma and 
timed urine samples are collected to determine the marker concentration (Urine Conc and Plasma Conc, $\mu \mathrm{mol} / \mathrm{L}$ ), and the rate of urine flow (Urine Flow, $\mathrm{mL} / \mathrm{min}$ ).

$$
\text { GFR }=\frac{\text { Urine Conc } \times \text { Urine Flow }}{\text { Plasma Conc }}
$$

In practice, this technique has limitations, as it is difficult and slow to achieve a steady state. In contrast, bolus methods can be quickly carried out, and require only a single injection of the exogenous marker into the plasma, and serial blood sampling at defined intervals. Urine collection is not required. However, data analysis requires the application of a well defined mathematical model. These exogenous marker studies are used primarily in research, and are the research gold-standard assessment.

\subsection{Endogenous Markers for measuring GFR}

Measuring GFR via the clearance of an exogenous marker (e.g. inulin) is time consuming, expensive, and not appropriate in most clinical settings. Hence, GFR is usually estimated using an endogenous marker, such as creatinine.

A previously used standard method of measuring GFR, is the creatinine clearance utilizing a 24 hour urine collection method. Using urinary and plasma creatinine, Equation (1) allows calculation of the creatinine clearance. While this overestimates GFR by $10 \%$ or more because of tubular secretion of creatinine [9], it nevertheless allows an approximation of GFR. Short collection periods can be useful [10, 11], but their use remains controversial. 24 hour collections are also often flawed because of collection problems [6]. 
Steady state serum creatinine is approximately inversely proportional to GFR, and hence GFR can be estimated without the requirement for a 24 hour urine collection. However, creatinine is not an ideal filtration marker because, in addition to being filtered by the glomerulus, it is also secreted by the proximal tubules, resulting in a clearance that exceeds GFR, particularly in ARF.

Since creatinine generation is determined mainly via muscle mass and dietary intake, tubular secretion of creatinine depends on factors such as age, sex, race, body size and muscle mass, and can vary substantially between individuals and over time [7]. Equations such as the Cockcroft-Gault and the MDRD equations have been developed to try to correct for these limitations [12]. Additionally, extra-renal elimination of creatinine via intestinal bacteria can increase at low GFR, thus making the method misleading for those individuals outside the normal range of kidney function. Finally, in ARF plasma creatinine levels are very slow to react to changes in GFR, because of the associated increase in creatinine half-life. With a 5 fold reduction in GFR, the creatinine half-life would increase from a normal value of 4 hours in man, to 20 hours. With 3-5 half lives required to reach a steady state, such a reduction in GFR would take approximately 80 hours to reach a new steady state. Until a new steady state is reached, the severity of the decrease in GFR cannot be ascertained by a serum creatinine measurement. The greater the reduction in GFR, the longer this assessment will take. Hence, these methods are a useful clinical tool due to their ease, but may have significant error. 


\subsection{SIFT-MS Breath Sampling}

SIFT-MS is a quantitative mass spectrometric method that utilizes the chemical ionization of positively charged precursor ions that react with the VOCs in an air or breath sample introduced into a flow tube. Comparing the mass of the product ions in a database, the identity of the sample VOCs can be established [13]. Changes in VOC concentration over time can provide an indication of disease progress. In this study, ammonia is the VOC of interest, since blood and breath ammonia increase in impaired renal function.

\subsection{Summary}

Overall, this paper compares 3 methods of renal function assessment. SIFT-MS is the new potential and non-invasive method. In contrast, creatinine clearance and exogenous inulin bolus are existing clinical and research standards. The outcome of this comparison will thus indicate both the differences in test accuracy or resolution, and the clinical applicability or context. 


\section{Methodology}

\subsection{Experimental Method}

Female Sprague-Dawley rats $(280-320 \mathrm{~g}, \mathrm{n}=8)$ were housed at $21^{\circ} \mathrm{C}$ in a 12 hour light/dark cycle, and allowed free access to food and water. Under ketamine/domitor anaesthesia, an in-dwelling cannula was inserted into the jugular vein for the purposes of fast serial blood sampling. GFR was monitored via plasma creatinine, SIFT-MS breath sampling, and bolus inulin clearance for 5 days while the animal recovered from surgery. In a subsequent surgery, the renal arteries were exposed via a single mid-ventral incision, and clamped for 60 minutes, creating ARF via an ischemic event. GFR was monitored for 7 days using the same 3 techniques, as the animal recovered and renal function returned to normal levels. The GFR monitoring schedule is shown in Table 1.

Table 1. GFR Monitoring Schedule

\begin{tabular}{|c|c|c|}
\hline \multirow{2}{*}{ Method } & \multicolumn{2}{|c|}{ Monitoring Time Points } \\
\hline & Post-Cannulation & Post-ARF \\
\hline Plasma & 6hr, 2day & 1hr, 6hr, 20hr, 30hr, 2day, \\
\hline Creatinine & & 4day, 7day \\
\hline $\begin{array}{c}\text { Inulin } \\
\text { Clearance }\end{array}$ & 6hr, 2day & 6hr, 30hr, 2day, 4day, 7day \\
\hline SIFT-MS & -4hr, 1hr, 6hr, 20hr, & -4hr,1hr, 6hr, 20hr, 30hr, \\
\hline Breath & 30hr, 2 day, 5 day & 2day, 3day, 4day,5day, 7day \\
\hline
\end{tabular}




\subsection{Radio-labelled Inulin Clearance}

${ }^{14} \mathrm{C}$ labelled inulin $(1 \mu \mathrm{Ci}$ of $1.12 \mu \mathrm{Ci} / \mathrm{mg}$ dissolved in $0.1 \mathrm{~mL}$ ionised water, diluted 1:4 with saline) was injected into the jugular vein cannula of a conscious animal over 2 seconds, and flushed with heparinised saline. A $150 \mu \mathrm{L}$ blood sample was collected via the jugular vein cannula at $-1,1.5,3,6,12,20,40,70$ minutes post-bolus, and sent for scintillation analysis. At 1 minute prior to injecting the bolus, an additional $150 \mu \mathrm{L}$ blood was collected for creatinine analysis. Red cells and remaining blood plasma were returned to the animal via the jugular vein after each experiment.

\subsection{Breath Sampling via SIFT-MS}

Direct breath was collected from conscious rats by wrapping the rat in a towel and placing it into a 300mL bottle (Fig. 2).

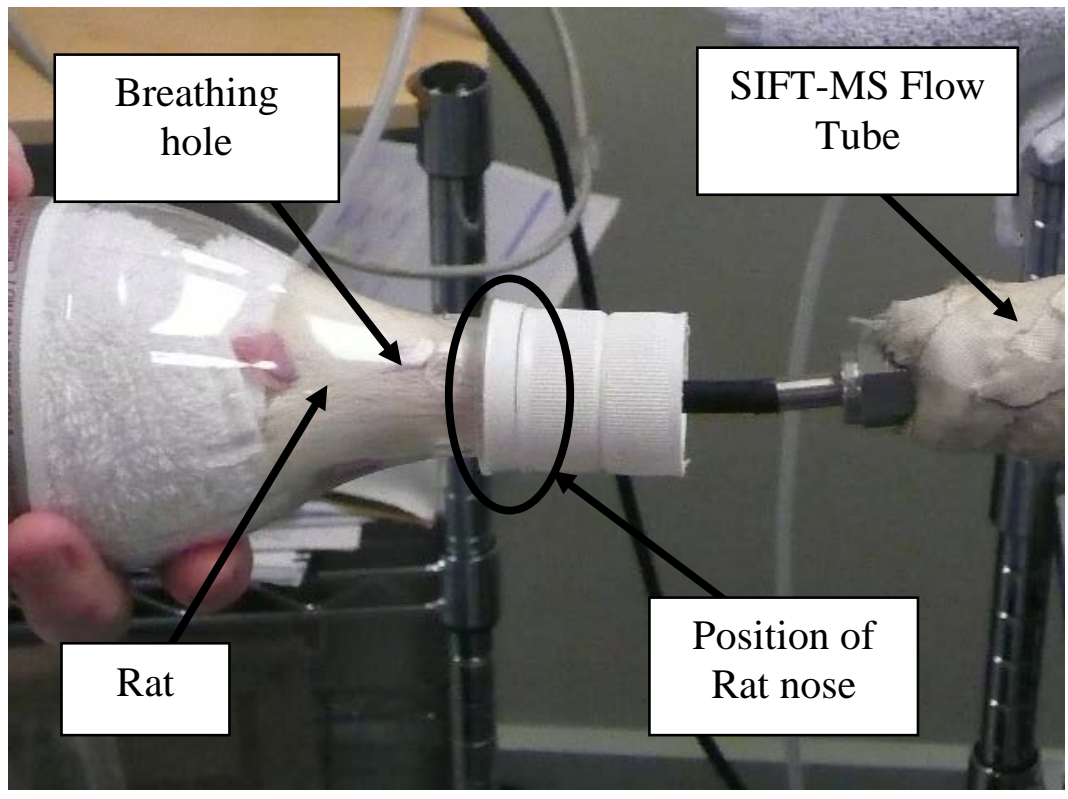

Fig. 2. Breath Collection Apparatus 
A background air sample was also obtained at each rat breath sample time-point. The tightness of the fit in the bottle combined with wrapping the rat in a towel minimized contamination from the fur and waste products. Breathing holes were located posterior to the position of the rats' nose. Breath was drawn directly from the bottle through the SIFT-MS machine at $2 \mathrm{~mL} / \mathrm{sec}$ via a small straw attached to the end of the bottle. Because rats of this size have a breath flow rate of $2-3 \mathrm{~mL} / \mathrm{sec}$, background air plays a significant role.

Excretion and uptake of VOCs in the breath depends on the ventilation/perfusion ratio in the lung, and the concentration gradients of the substances across the respiratory surface and between the alveolar and environmental gas. Therefore, a subject breathing in an environment with a low VOC concentration will measure different alveolar VOC concentrations to the same subject breathing in a high VOC environment because of the different concentration gradient for diffusion. Similarly, when the external VOC concentration becomes higher than the endogenous concentration, the VOC will diffuse into blood instead of appearing in the exhaled breath.

The low breath flow rate of rats, combined with the relatively high sampling rate of the Voice100 SIFT-MS machine, means that a highly mixed sample made up of the background air as well as the (background-air-dependent) alveolar breath, was tested. Most studies use mixed expiratory sampling, which includes deadspace air and hence artificially dilutes (for endogenous substances), or concentrates (for external contaminants) the measured analyte. However, if background air is also sampled, and 
the deadspace volume can be accounted for, the alveolar fraction of the breath can be extracted [14-17].

The most common method for breath testing in large animals is via a face-mask with a non-rebreathing valve, and collecting the breath exhalate into a bag. In small animals, the animal is usually placed in a sealed chamber until desired analytes reach detectable levels. In anaesthetised animals, breath is usually collected directly using endotracheal intubation [18]. In this study, direct breath was deemed preferable to breath collection in tedlar bags due to the difficulty in remote collection of ammonia [19]. Endotracheal intubation was not considered for the primary breath sampling method in the main trial because it requires the animal to be anaesthetised. Due to the number of breath samples required, this method was deemed impractical, considering the risks of repeated anaesthesia, and animal ethics.

The option to place the animal in a sealed chamber, and wait for analyte concentrations to build up was discarded for two main reasons. Firstly, to simply wait for detectable analyte levels to be obtained is inefficient, since the analyte will continue to build up after alveolar gas concentrations are reached. To wait for this pseudo-equilibrium would be dangerous for the rat, since it involves re-breathing and thus concentrating $\mathrm{CO}_{2}$ in the blood and risking hypercapnia. Secondly, the sample collected would be contaminated by fur and waste products, for example if the animal urinated whilst in the chamber. 
The face-mask option with re-breathing valve commonly used for breath sampling in large animals was attempted for the rat. A latex mask was attached to straw with a simple reed valve, allowing the rat to breath from the room air, and forcing breath unidirectionally down the straw to the mass spectrometer. Due to the low pressures involved because of the small size of the animal, combined with the difficulty in forming a seal around the mask, activating the valves was very difficult. In addition, because of the small tidal volume, deadspace was a significant problem. Lastly, it was very stressful for the rat to be forced into the mask, likely elevating stress markers, such as pentane in the breath. Valved methods such as utilised in humans for discarding the dead-space portion of the breath and maintaining the alveolar breath for sampling were also discarded as impractical in the rat. 


\section{Mathematical Models}

\subsection{Inulin Clearance}

Injection of an inulin bolus into the blood is described by the 2-compartment model in Fig. 3, where the bolus is injected into the plasma compartment, $P$, (with distribution volume $V_{P}$ ), and moves bi-directionally between the interstitial compartment, $Q$, (distribution volume $V_{Q}$ ), as described by $n_{2}$ and $n_{3}$ before being eliminated from the plasma via the kidneys, as described by $n_{1}$.

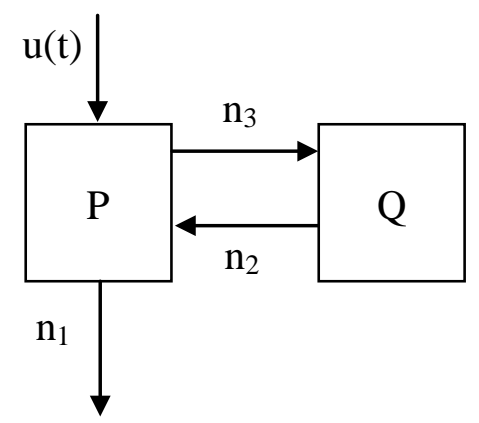

Fig. 3. Two-Compartment Model of Kidney Function

The differential equations describing the amount of inulin in the 2 compartments are dependent on the three rate constants $n_{1}-n_{3}$, and the inulin mass in both compartments, $p(t)$ and $q(t)$, as described in Equations (2) and (3).

$$
\begin{gathered}
\frac{d}{d t} p(t)=-\left(n_{1}+n_{3}\right) p(t)+n_{2} q(t)+u(t) \\
\frac{d}{d t} q(t)=-n_{2} q(t)+n_{3} p(t)
\end{gathered}
$$

In terms of concentrations, where $P(t)=p(t) / V_{P}$ and $Q(t)=q(t) / V_{Q}$, and defining $\alpha=$ $V_{Q} / V_{P}$, Equations (2) and (3) become: 


$$
\begin{gathered}
\dot{P}(t)=-\left(n_{1}+n_{3}\right) P(t)+n_{2} \alpha Q(t)+\frac{u(t)}{V_{P}} \\
\dot{Q}(t)=-n_{2} Q(t)+\frac{n_{3}}{\alpha} P(t)
\end{gathered}
$$

Since transport is assumed to be passive between plasma and interstitial compartments, the volume flowrate between compartments must be equal, indicating a diffusion-based transport, as defined in Equation (6).

$$
n_{3} V_{P}=n_{2} V_{Q}
$$

It is assumed that the bolus is given so quickly that the concentration in the interstitial compartment, $Q(t)$, is initially zero (as verified by the -1 minute blood sample). The bolus is therefore modelled as a unit impulse function at time zero, as described in Equation (7), where $P(0)$ is the inulin concentration in the plasma compartment at time zero, and $\delta(t)$ is the Dirac delta function.

$$
u(t)=P(0) V_{P} \delta(t)
$$

Equation (7) is a reasonable simplification given there is no extra information to be obtained by modelling the initial rise in plasma inulin concentration, giving the physical limitations of gaining a sufficient number of accurate sample datapoints within the first minute after injection of the bolus. This assumption also indicates that the initial concentration in the plasma compartment is determined from the size of the injected bolus and the plasma volume. Combining Equations (4), (5), (6) and (7) yields: 


$$
\begin{gathered}
\dot{P}(t)=-n_{1} P(t)-n_{2} \alpha(P(t)-Q(t))+P(0) \delta(t) \\
\dot{Q}(t)=n_{2}(P(t)-Q(t))
\end{gathered}
$$

Equations (8) and (9) can be solved by standard non-linear regression [20]. However, as with any non-linear regression there can be problems with the existence of local minima. In addition, it can be computationally expensive due to the requirement for extensive forward simulations of the model. A detailed analysis of this phenomenon in a similar model is given in [21]. The method proposed is an extension of the integral method in [21] and is similar to the approach in [22]. It is very fast computationally.

The method starts by integrating Equation (8) to obtain an approximation to $P(t)$ in terms of the unknowns $n_{1}$ and $n_{2}$. A system of equations is then set up as defined in Equation (10), and solved for $n_{1}$ and $n_{2}$ using linear least squares.

$$
\left[\int_{0}^{t} P(t) d t \quad \alpha \int_{0}^{t}(P(t)-Q(t)) d t\right]\left[\begin{array}{l}
n_{1} \\
n_{2}
\end{array}\right]=[P(0)-P(t)]
$$

Note that Equation (10) will have to be integrated over several different time intervals in the test to yield a solvable system of equations. The initial approximation of $P(t)$ is a linear piecewise approximation obtained from the measured time and concentration data, and $P(0)$ is the estimated average inulin concentration in the plasma immediately after injection of the bolus, defined as the product of the bolus volume and concentration, divided by the estimated plasma volume of the animal based on its weight. Using the obtained solution for $n_{1}$ and $n_{2}$, a new approximation for $P(t)$ is 
generated. Constraints are added to the least squares solution to keep the approximation within known physiological ranges. However, there are no measurements for $Q(t)$, hence a different approach is required to analyse the interstitial compartment.

Given the approximation for $P(t), Q(t)$ can be found analytically using the convolution integral solution to Equation (9), given in (11).

$$
Q(t)=n_{2} \int_{0}^{t} P(\tau) e^{-n_{2}(t-\tau)} d \tau
$$

Iterations between $P(t)$ and new estimates of $Q(t)$ to update $P(t)$ in Equation (10) continue until convergence is achieved in the parameter values. The method is repeated for $\alpha$ values within the physiologically reported range of 2.2 - 4.5 [23, 24], until a least squares solution is found for $\alpha$.

\subsection{Plasma Creatinine}

The Cockcroft-Gault equation for GFR in humans is:

$$
\text { Creatinine Clearance }=\frac{(140-\text { age })(w t)(0.85 \text { if female })}{72 C r}
$$

where age is patients age (years), wt is their mass (kg), and $\mathrm{Cr}$ is the plasma creatinine concentration $(\mathrm{mg} / \mathrm{dL})$. All animals in the present study were female and approximately the same age. Therefore, by plotting GFR (mL/min) values generated 
using the inulin clearance method against $\frac{\text { mass }}{C r}$ for all rats at all time points, Fig. 4 and Equation (13) were obtained, with a Pearson product-moment correlation coefficient (R-value) of $\mathrm{R}=0.94$.

$$
G F R=220 \times 10^{-3} \mu \mathrm{mol} / \mathrm{min} / \mathrm{kg} \times \frac{\text { mass }}{\mathrm{Cr}}
$$

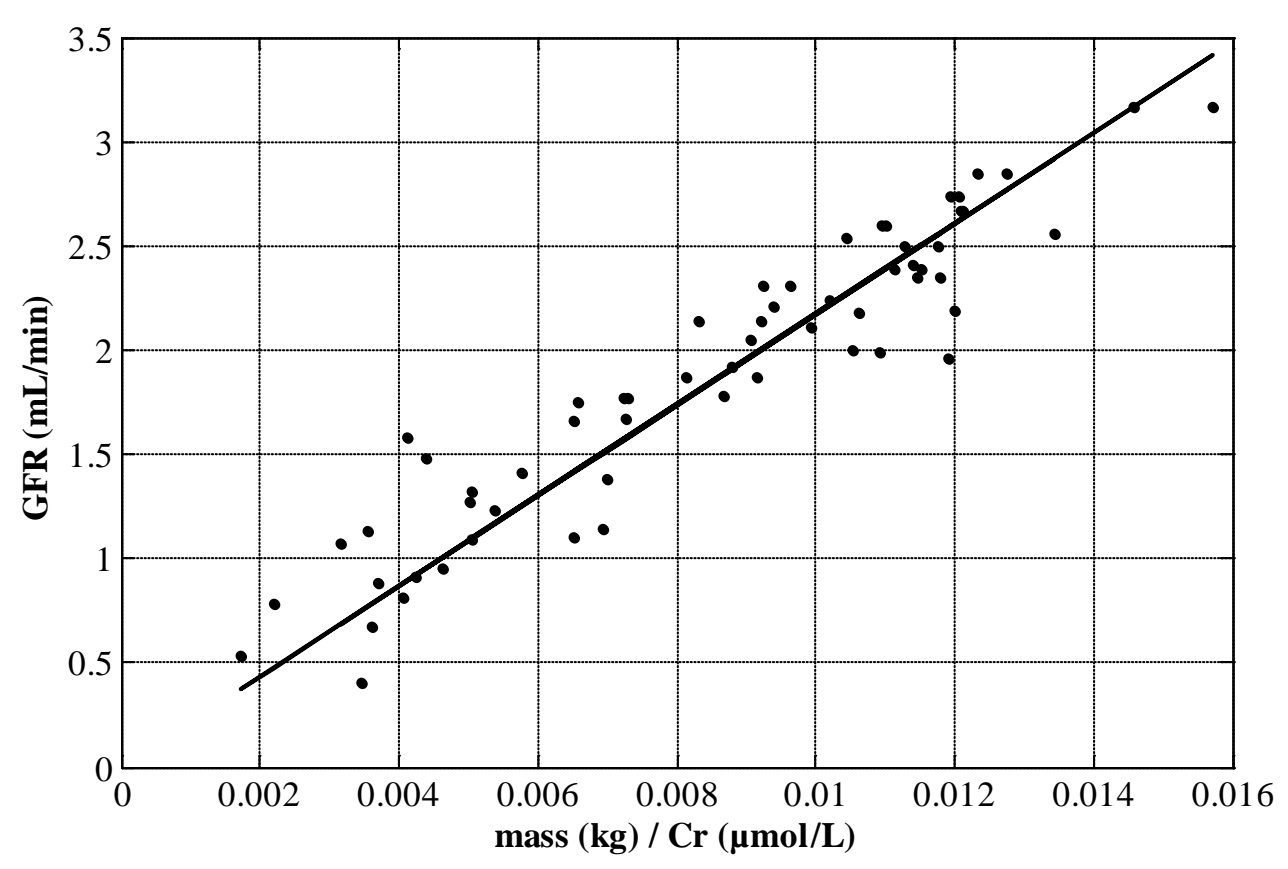

Fig. 4. Relationship between Creatinine, Mass and GFR

\subsection{SIFT-MS Breath Sampling}

A pilot study was conducted to determine the relationship between rat breath and background air in the experimental set-up described in Section 2.2. Healthy, conscious rats were allowed to equilibrate in a room containing a controlled concentration of ammonia. By increasing the concentration of the background 
ammonia, $C_{B}$, and measuring the sample collected in the bottle, $C_{\text {Tot }}$, Fig. 5 was obtained.

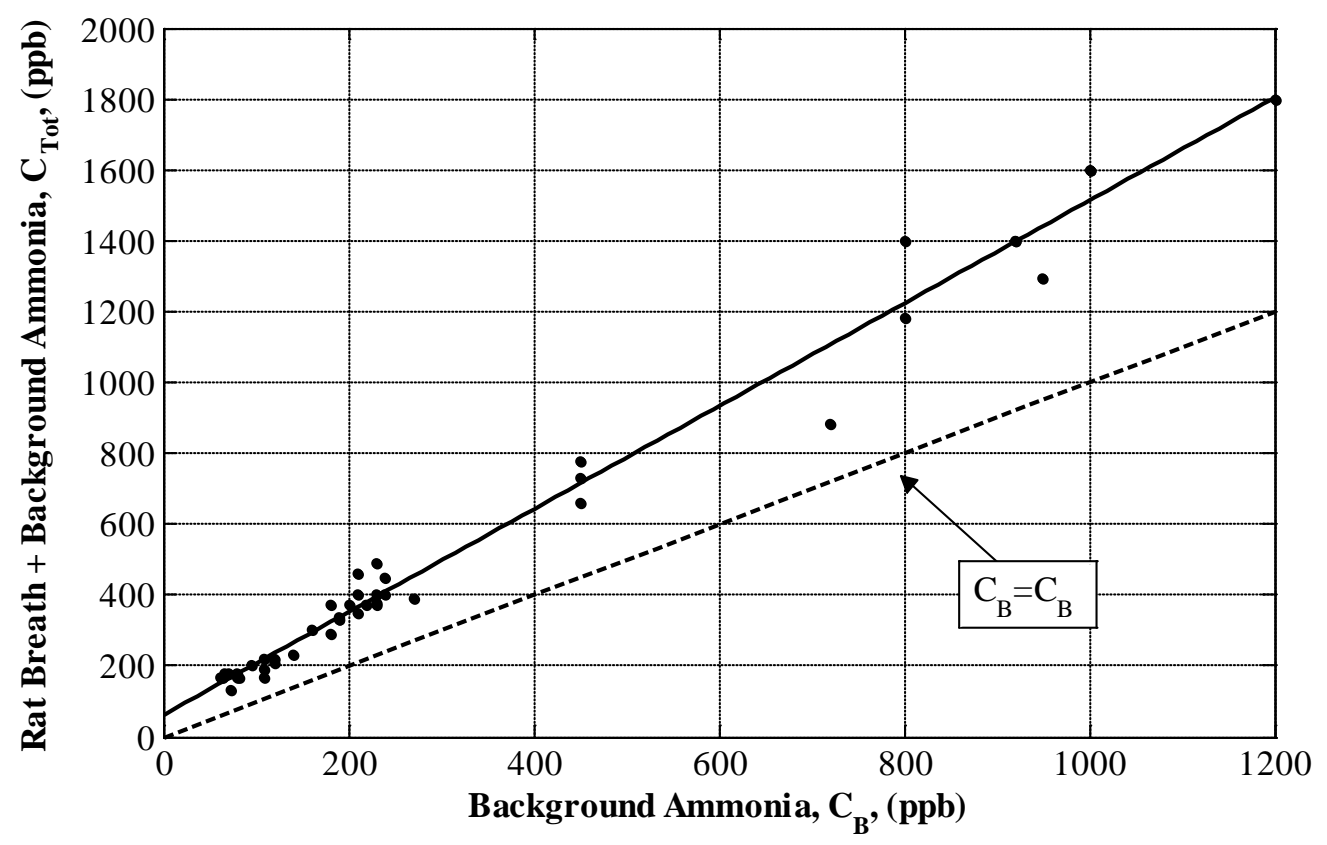

Fig. 5. Relationship between Background and Breath in healthy rats

Linear regression yields Equation (14), with an R-value of 0.99, where the x-intercept represents the concentration of the analyte in breath given a zero concentration in background air. The slope of $C_{\text {Tot }}$ greater than 1 indicates that ammonia was concentrated in the breath against its concentration gradient from the blood, an interesting, unique finding that has not been previously described.

$$
C_{\text {Tot }}=1.46 \times C_{B}+59 p p b
$$

For simple diffusion down a concentration gradient, as usually reported for transport across lung epithelial cells, it was expected that when plotted against $C_{B}$, the lines 
$C_{T o t}$ and $C_{B}$ should intersect at a point where the concentrations are equal. At background air concentrations below the point of intersection, the analyte diffuses out of the blood, and at background air concentrations greater than the point of intersection, the analyte diffuses into the blood. Hence, this finding in Fig. 5 and Equation (14) suggests the presence of actively energised ammonia transporters in the lung capillaries or epithelia, which are capable of transporting ammonia against a concentration gradient such that ammonia appears to be exhaled at concentration greater than that of the background air or the blood. Another possible explanation is that ammonia and its metabolites are removed from the blood and concentrated in the lung tissue, thus providing a sink for diffusion into the alveolar air. Cooper and Freed [25] injected a $\left[{ }^{13} \mathrm{~N}\right]$ ammonia bolus into the femoral vein of anaesthetised rats and measured its clearance from the blood. Results showed that nearly 30\% of the label was removed by the lungs in the first pass, supporting the theory for an ammonia sink. However, they did not measure large quantities of ammonia in the expired air. A third possible explanation is an increase in the action of the enzyme urease in the oral cavity, which converts urea to ammonia. However, this effect is expected to be less pronounced in rats since they are obligate nose breathers.

A lumped parameter model for the breath circuit is shown in Fig. 6, where the air/breath moving into and out of the bottle is considered. In this model, the rat is breathing at a volume flow rate of $V_{R} \mathrm{ml} / \mathrm{sec}$, and breathes in and out concentrations of $C_{T o t}$ and $C_{R}$, respectively. In Fig. 6, this corresponds to air leaving the bottle at a volume flow rate of $V_{R} \mathrm{ml} / \mathrm{sec}$ and concentration of $C_{T o t}$ as the rat breathes in, and breath entering the bottle at a volume flow rate of $V_{R} \mathrm{ml} / \mathrm{sec}$ and concentration of $C_{R}$ as the rat breathes out. SIFT-MS draws the sample at $2 \mathrm{~mL} / \mathrm{sec}$, and thus background 
air must be drawn into the bottle at $2 \mathrm{~mL} / \mathrm{sec}$ to maintain equilibrium. The volume flow rate of rat breath is calculated from tidal volume and respiratory rate, which for a 250-300g rat, is approximately $2.7 \mathrm{~mL} / \mathrm{sec}$ [26].

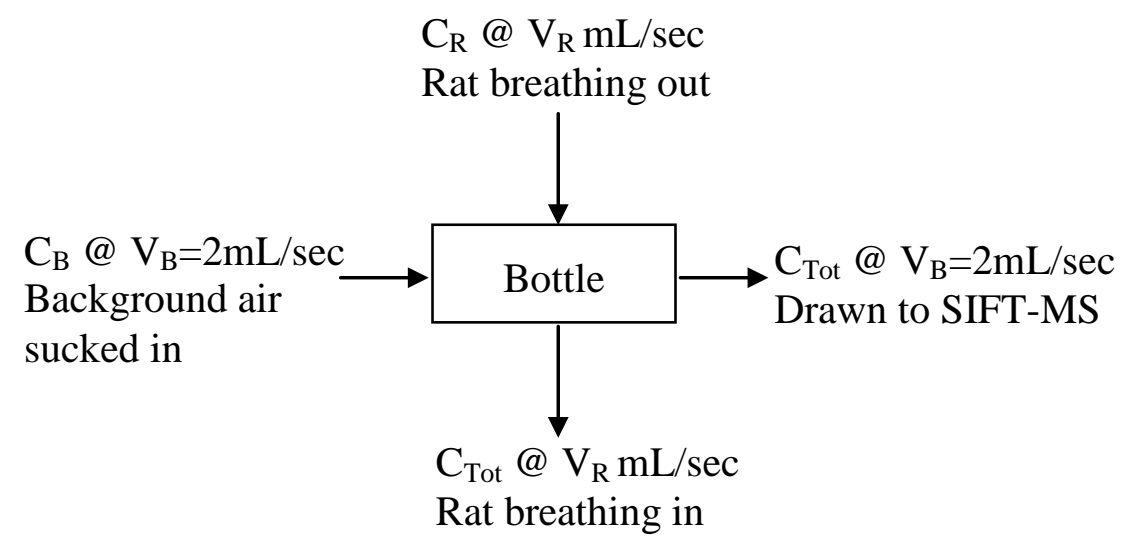

Fig. 6. Rat Breathing Circuit

Using conservation of mass, $C_{R}$ can be calculated:

$$
\begin{aligned}
& C_{B} V_{B}+C_{R} V_{R}=C_{T o t} V_{B}+C_{T o t} V_{R} \\
& \Rightarrow C_{R}=\left(1+\frac{V_{B}}{V_{R}}\right) C_{T o t}-\left(\frac{V_{B}}{V_{R}}\right) C_{B}
\end{aligned}
$$

Plotting $C_{R}$ against $C_{B}$ and fitting a linear regression, as in Fig. 5 and Equation (14), yields:

$$
C_{R}=1.79 \times C_{B}+102 p p b
$$


Note that the $C_{R}$ obtained from Equation (16) is specific for a healthy rat. Hence, Equations (15) and (16) are combined to normalise a given rat concentration, $C_{R}$, to a healthy rat concentration at that background air concentration, as seen in Equation (17).

$$
C_{R^{*}}=\frac{\left(1+\frac{V_{B}}{V_{R}}\right) C_{T o t}-\left(\frac{V_{B}}{V_{R}}\right) C_{B}}{102 p p b+1.79 C_{B}}
$$

$C_{R^{*}}$ was plotted against time, following the course of each surgical intervention. Hence, each rat provided their own control from the first surgery to ensure that it was the ARF and not surgery alone causing the rise in breath ammonia. Using this method, a percentage increase in breath ammonia can be reported for each rat.

Alternatively, breath ammonia can be converted to GFR by plotting GFR obtained via inulin clearance against $C_{R^{*}}$ for all rats at all time-points (Fig. 7). Hence, GFR can be estimated from breath ammonia using the linear regression of Equation (18), which results in a R-value of 0.89 . Note that domitor, used in the anesthetic cocktail, causes a 70\% depression in respiration. Therefore, the ratio $V_{B} / V_{R}$ increases during surgery and recovery from anesthesia. 


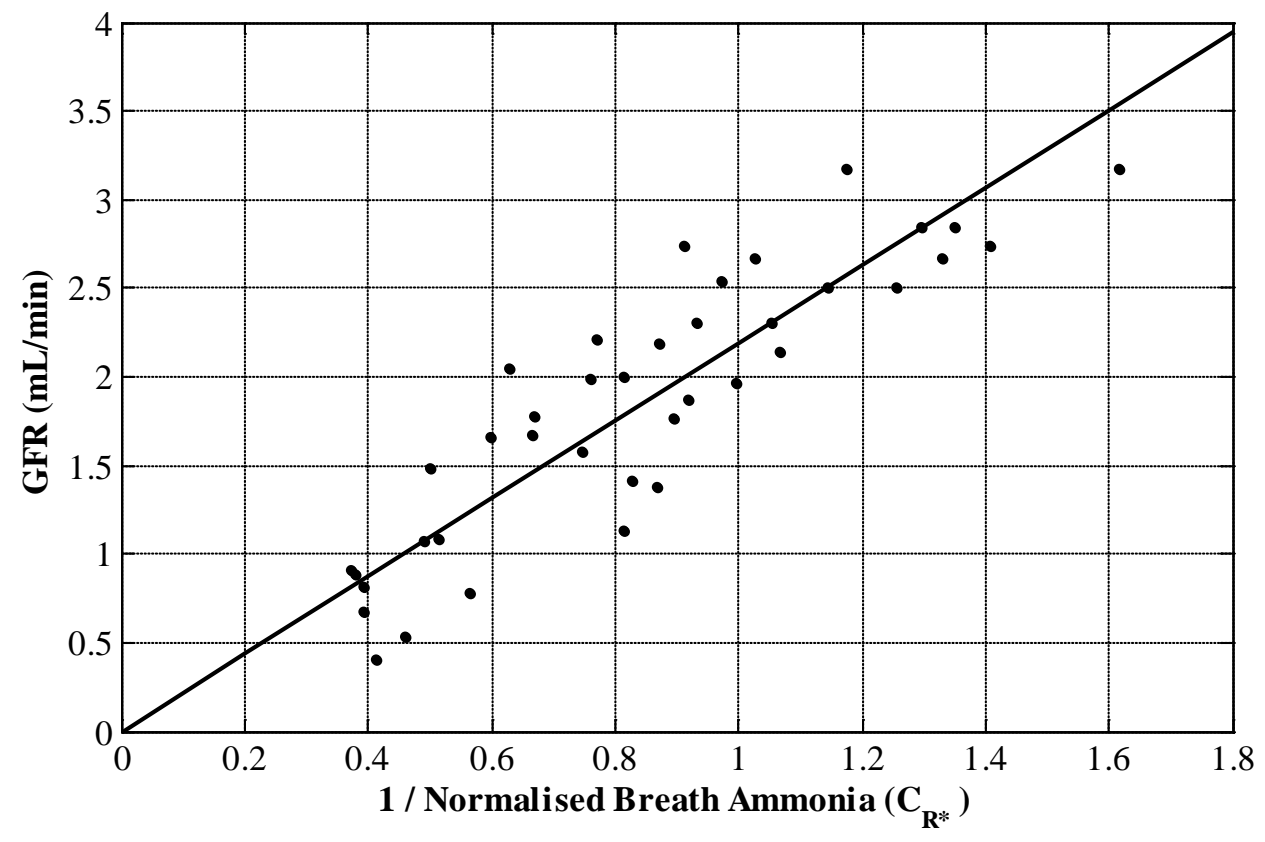

Fig. 7. Relationship between Breath Ammonia and GFR

$$
G F R=\frac{2.19 \mathrm{~mL} / \mathrm{min}}{C_{R^{*}}}
$$




\section{Results and Discussion}

\subsection{Bolus Radio-labelled Inulin Clearance Test}

Using the iterative integral fitting method, clearance curves were obtained for each rat at each time-point. Due to the difficulty in keeping the jugular vein cannula patent for 12 days, some animals did not undergo the full contingent of 7 inulin clearance tests. Fig. 8 shows a typical inulin clearance curve obtained for a rat in the normal and ARF states. The average curve fitting error was less than $10 \%$.

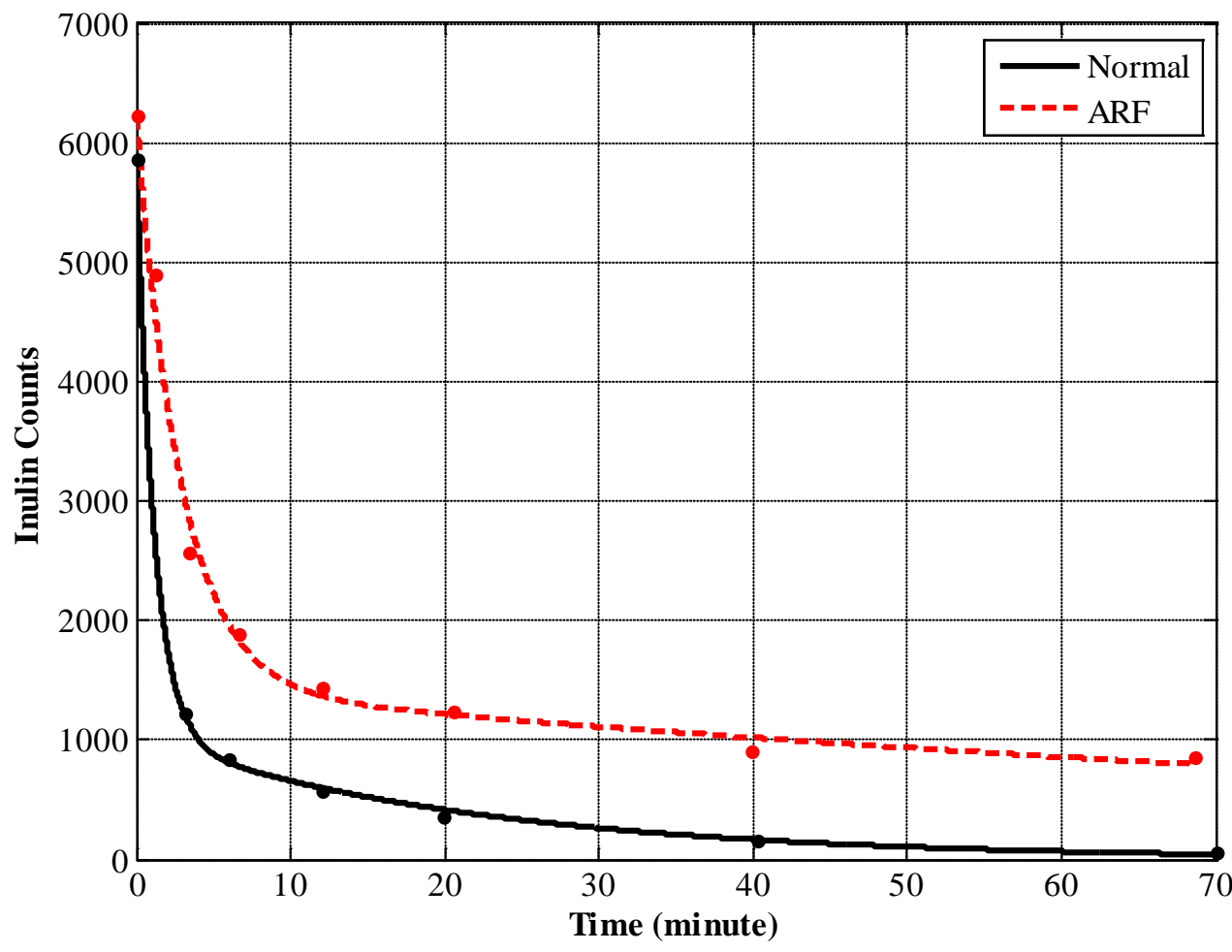

Fig. 8. Inulin Clearance Curve

Table 2 summarizes the inulin clearance test results. The maximum decrease in GFR was observed in the first clearance test post-ARF, at the 6 hour time-point. 
Table 2. Inulin GFR Estimation

\begin{tabular}{|c|c|c|c|c|c|}
\hline \multirow{2}{*}{ Rat } & \multirow{2}{*}{ Weight (g) } & \multicolumn{2}{|c|}{ GFR (mL/min)* } & \multirow{2}{*}{ \% decrease } & \multirow{2}{*}{$\begin{array}{c}\text { Model \% } \\
\text { error }\end{array}$} \\
\hline & & Normal & ARF & & \\
\hline 1 & 299 & 2.60 & 0.95 & 63 & 8.2 \\
\hline 2 & 300 & 2.50 & 0.40 & 84 & 7.3 \\
\hline 3 & 300 & 2.67 & 0.81 & 70 & 9.1 \\
\hline 4 & 302 & 2.39 & 1.1 & 54 & 9.1 \\
\hline 5 & 291 & 2.74 & 0.91 & 67 & 8.9 \\
\hline 6 & 280 & 2.35 & 1.05 & 55 & 10.9 \\
\hline 7 & 306 & 3.17 & 1.09 & 66 & 8.4 \\
\hline 8 & 312 & 2.86 & 0.68 & 76 & 7.3 \\
\hline
\end{tabular}

\subsection{Plasma Creatinine}

Maximum changes in plasma creatinine concentrations were found to occur between 6 and 20 hours post induction of ARF, as indicated in Table 3. Estimated GFR values obtained from Equation (13) are shown in Table 3, where the fitting factor refers to the constant in Equation (13) when data is fitted in a rat-specific manner, as opposed to across the tested rat population. This deviation in fitting factor gives some indication of the expected error in the GFR estimation when compared to the inulinclearance estimated GFR. 
Table 3. Creatinine GFR Estimation

\begin{tabular}{|c|c|c|c|c|c|}
\hline \multirow{2}{*}{ Rat } & \multicolumn{2}{|c|}{ GFR (mL/min)* } & \multirow{2}{*}{$\%$ decrease } & \multirow{2}{*}{$\begin{array}{l}\text { Fitting factor } \\
(\mu \mathrm{mol} / \mathrm{min} / \mathrm{kg})\end{array}$} & \multirow{2}{*}{$\begin{array}{l}\text { Hours to max. } \\
\text { conc. }\end{array}$} \\
\hline & Normal & ARF & & & \\
\hline 1 & 2.59 & 1.09 & 58 & 235 & 6 \\
\hline 2 & 2.56 & 0.33 & 87 & 217 & 20 \\
\hline 3 & 2.69 & 0.90 & 67 & 222 & 6 \\
\hline 4 & 2.49 & 1.21 & 51 & 243 & 20 \\
\hline 5 & 2.63 & 0.92 & 65 & 210 & 6 \\
\hline 6 & 2.43 & 1.01 & 58 & 209 & 6 \\
\hline 7 & 3.25 & 1.05 & 68 & 207 & 6 \\
\hline 8 & 2.78 & 0.78 & 72 & 212 & 6 \\
\hline
\end{tabular}

\subsection{SIFT-MS Breath Analysis}

Selected Ion Mode (SIM) scans for ammonia were performed on rat breath samples, and converted to GFR estimates as summarized in Table 4. The decreases (\%) in renal function results show excellent correlation with the inulin estimations of Table 2, with a Pearson product-moment correlation coefficient of 0.89 . 
Table 4. Breath Ammonia Determination of GFR

\begin{tabular}{ccccc}
\hline \multirow{2}{*}{ Rat } & \multicolumn{2}{c}{ GFR (mL/min)* } & \% decrease & Hours to max. \\
\cline { 2 - 5 } & Normal & ARF & & conc. \\
\hline 1 & 2.56 & 1.09 & 57 & 6 \\
\hline 2 & 2.75 & 0.42 & 85 & 6 \\
\hline 3 & 2.92 & 0.86 & 71 & 6 \\
\hline 4 & 2.35 & 1.17 & 50 & 6 \\
\hline 5 & 3.08 & 0.82 & 73 & 6 \\
\hline 6 & 2.31 & 1.21 & 48 & 6 \\
\hline 7 & 3.13 & 1.44 & 54 & 6 \\
\hline 8 & 2.96 & 0.85 & 71 & 6 \\
\hline $6 F$ & & & & 6 \\
\hline
\end{tabular}

* GFR calculated from Breath ammonia concentration (Equation 18)

Error in the GFR estimation is due to the oscillation in ammonia concentration as recorded by the SIFT-MS, and seen in Fig. 9. Fig. 9 shows the ammonia concentration as recorded by the SIFT-MS machine. The machine starts recording just before the sample is injected to obtain a background concentration, and then it takes approximately 20s to reach an equilibrium value. A probability density function can be formed around the concentration data, to obtain the inter quartile range of ammonia concentration. Because of the similar flowrates between breath and machine sampling, the error is effectively amplified in the current study when $C_{T o t}$ and $C_{B}$ are combined to obtain $C_{R}$. However, with the introduction of a new Voice $200^{\circledR}$ SIFT- 
MS machine, which samples at the lower rate of $2 \mathrm{~mL} / \mathrm{min}$ (as opposed to $2 \mathrm{~mL} / \mathrm{sec}$ ), this error could be minimized in future studies.

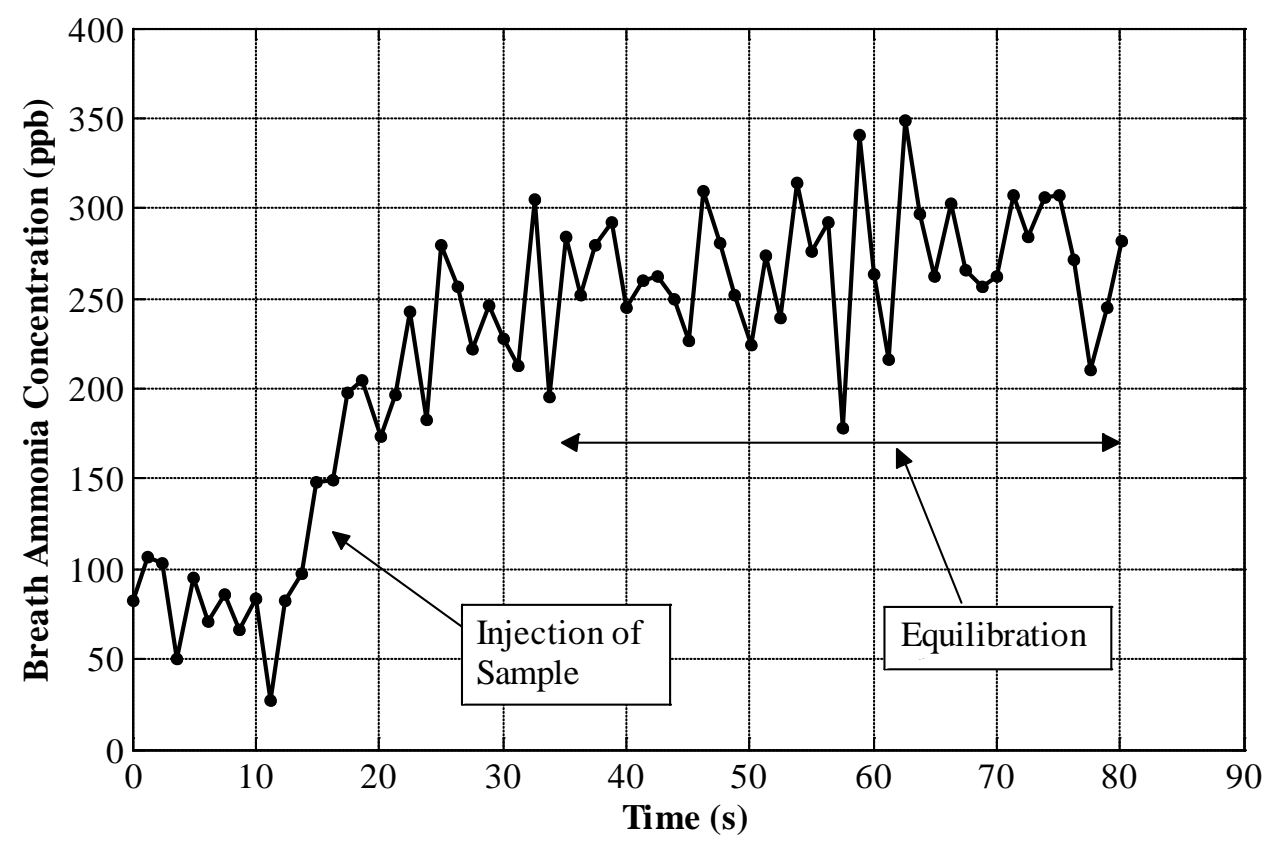

Fig. 9. Oscillation in Ammonia SIM Scan

\subsection{Comparison in GFR Estimations}

Very good correlations were observed between the percentage decreases in renal function estimated by the 3 different methods. When the population metrics of Equation (13) and Equation (18) were combined for the plasma creatinine and breath ammonia data to obtain GFR estimates, Figs. 10-17 were obtained for the 8 rats. 


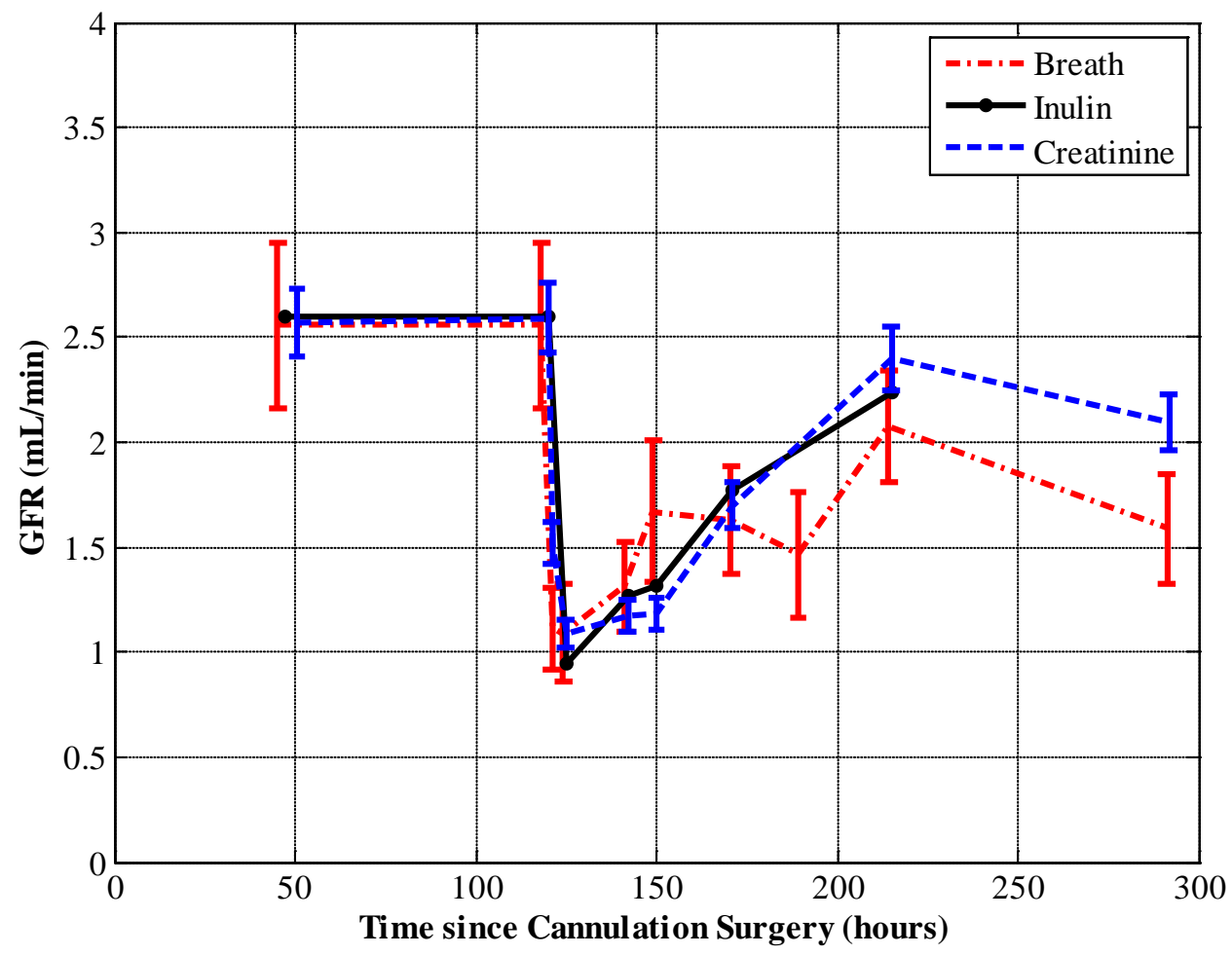

Fig. 10. Rat 1 GFR Comparision

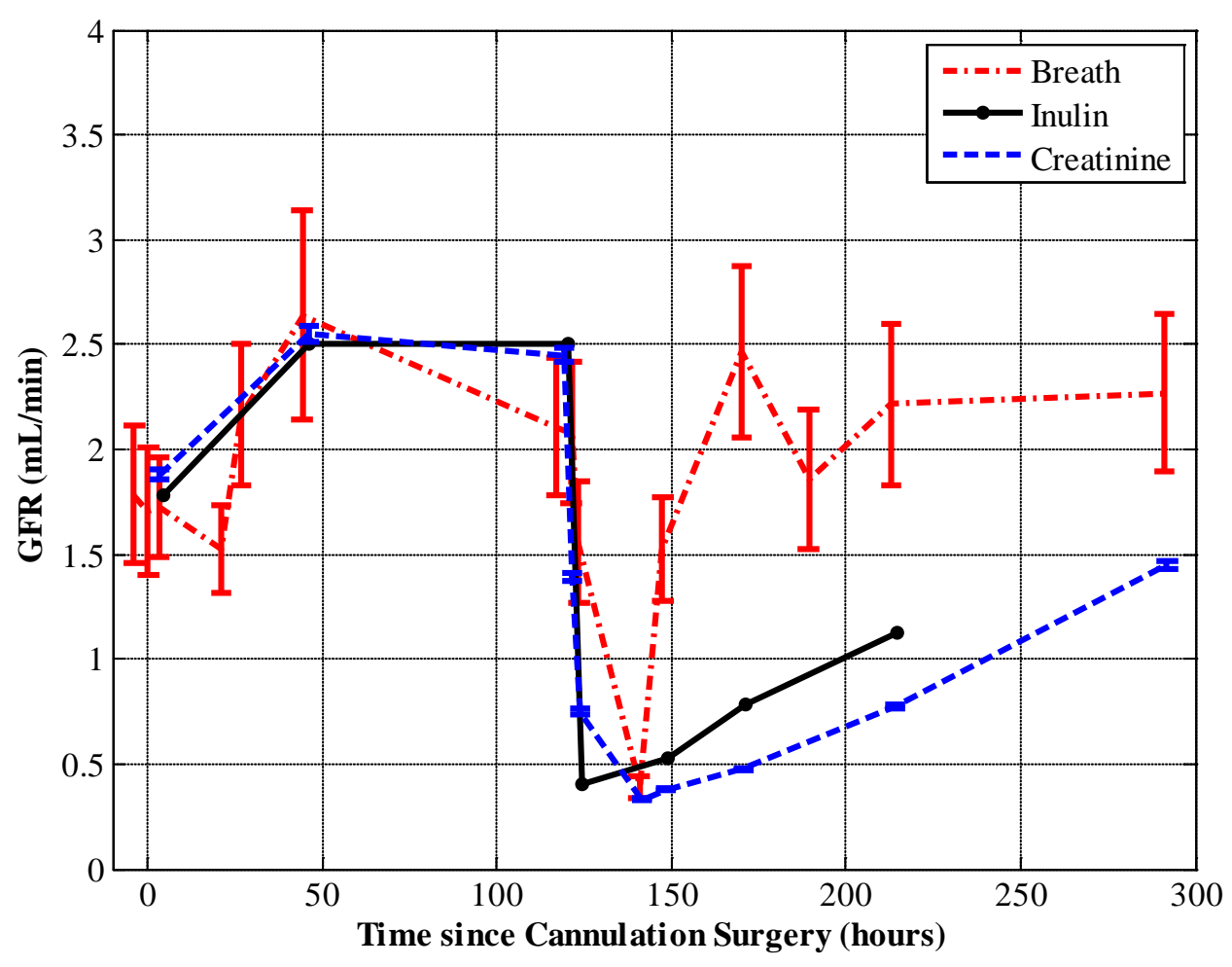

Fig. 11. Rat 2 GFR Comparision 


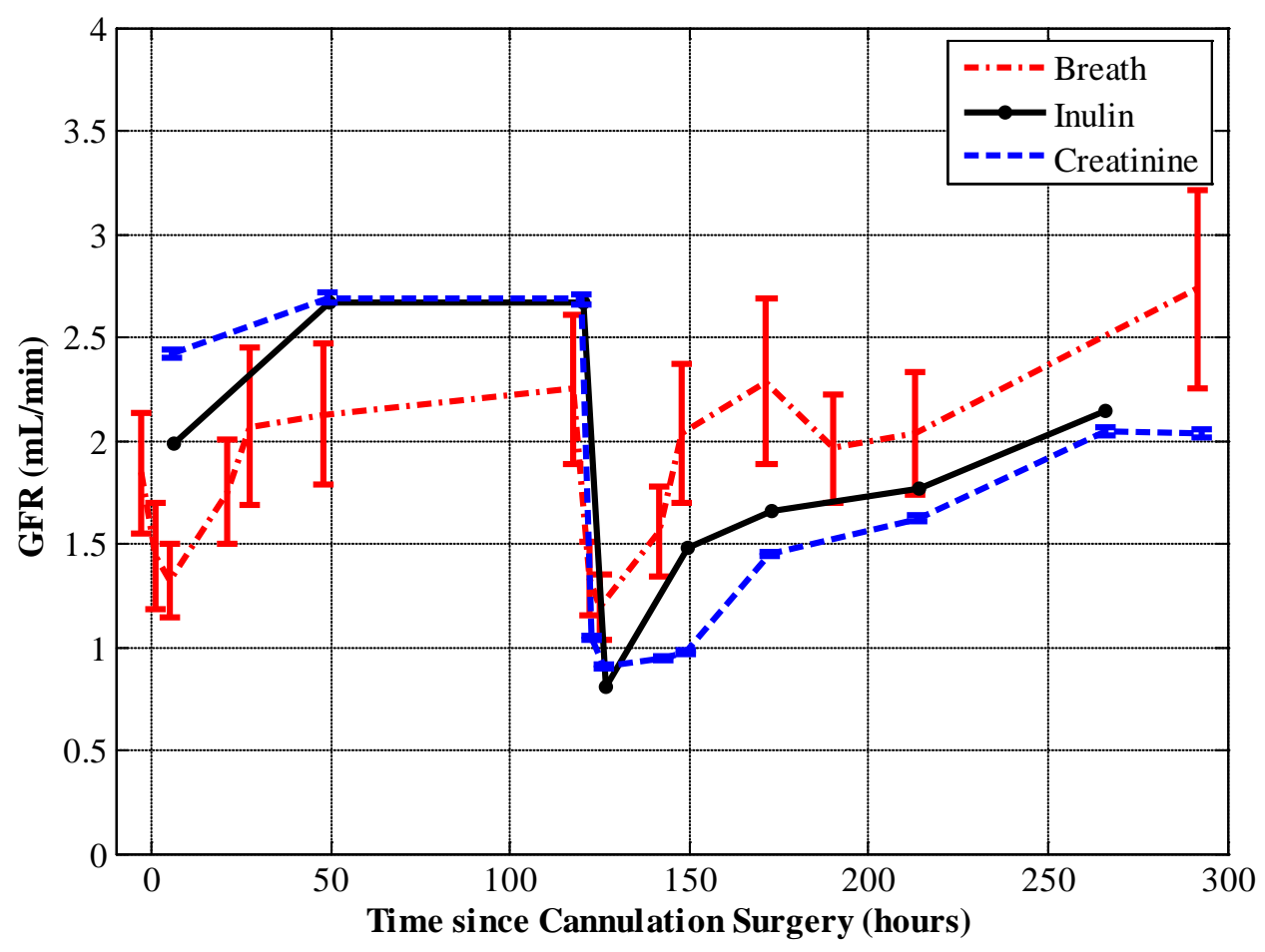

Fig. 12. Rat 3 GFR Comparision

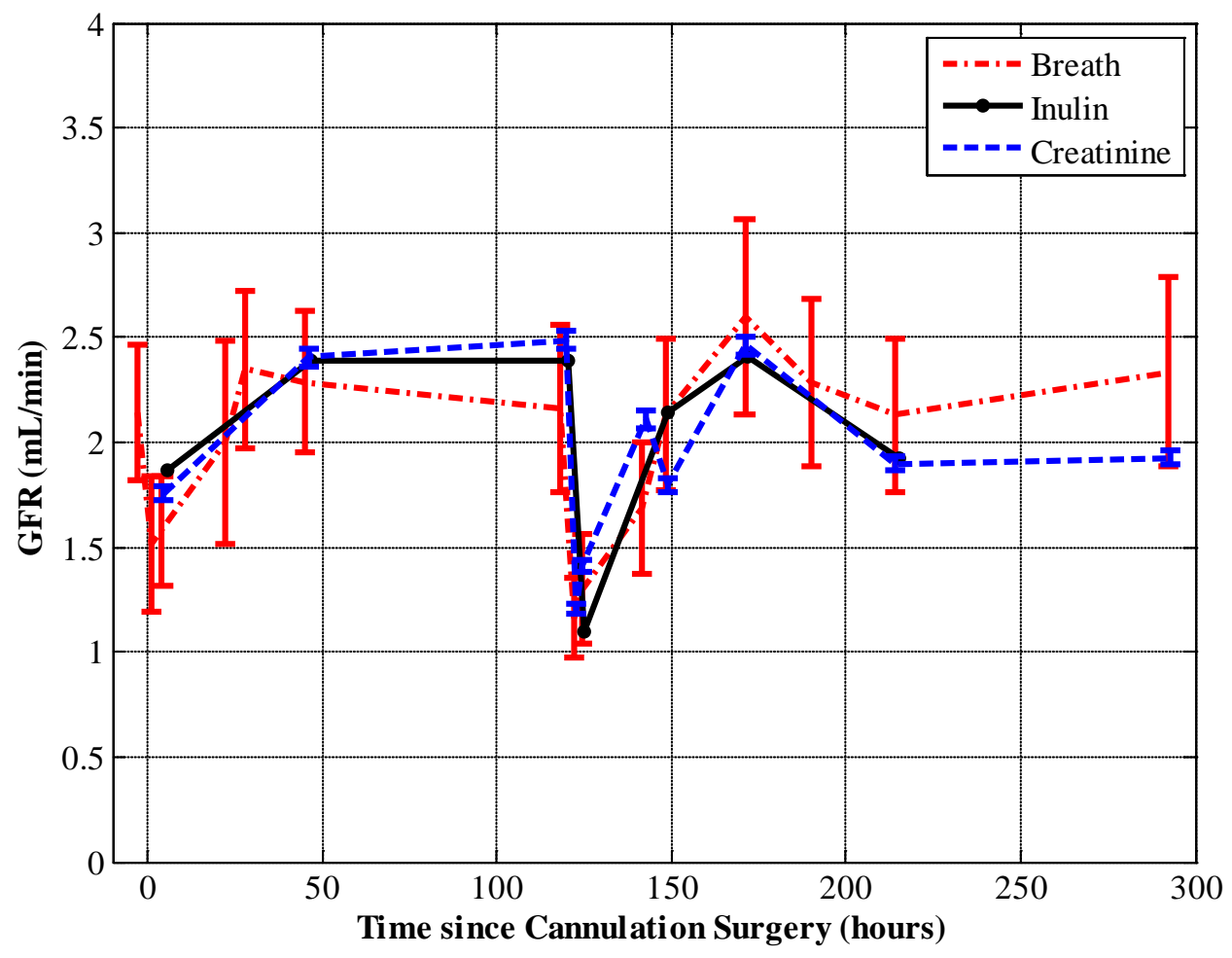

Fig. 13. Rat 4 GFR Comparision 


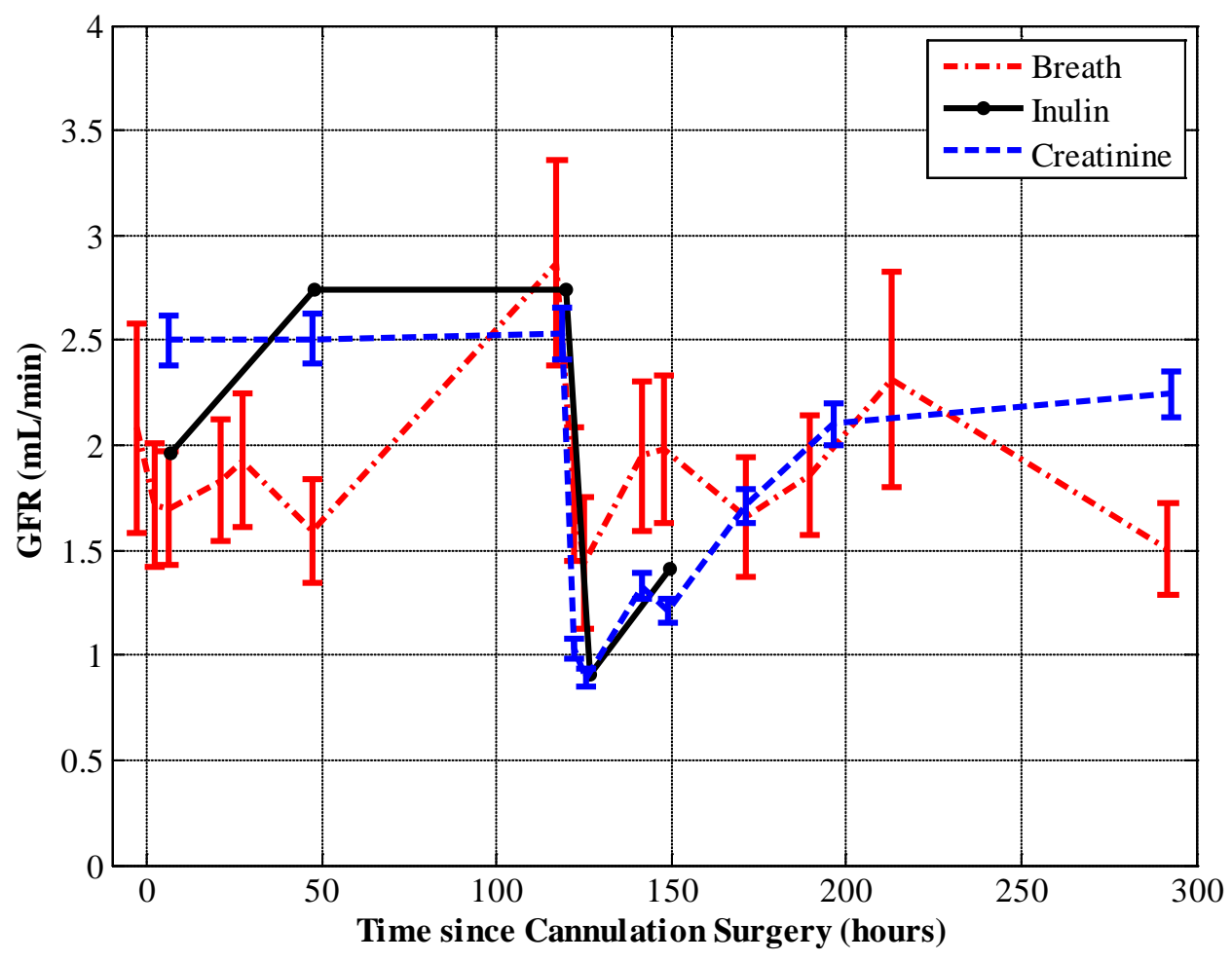

Fig. 14. Rat 5 GFR Comparision

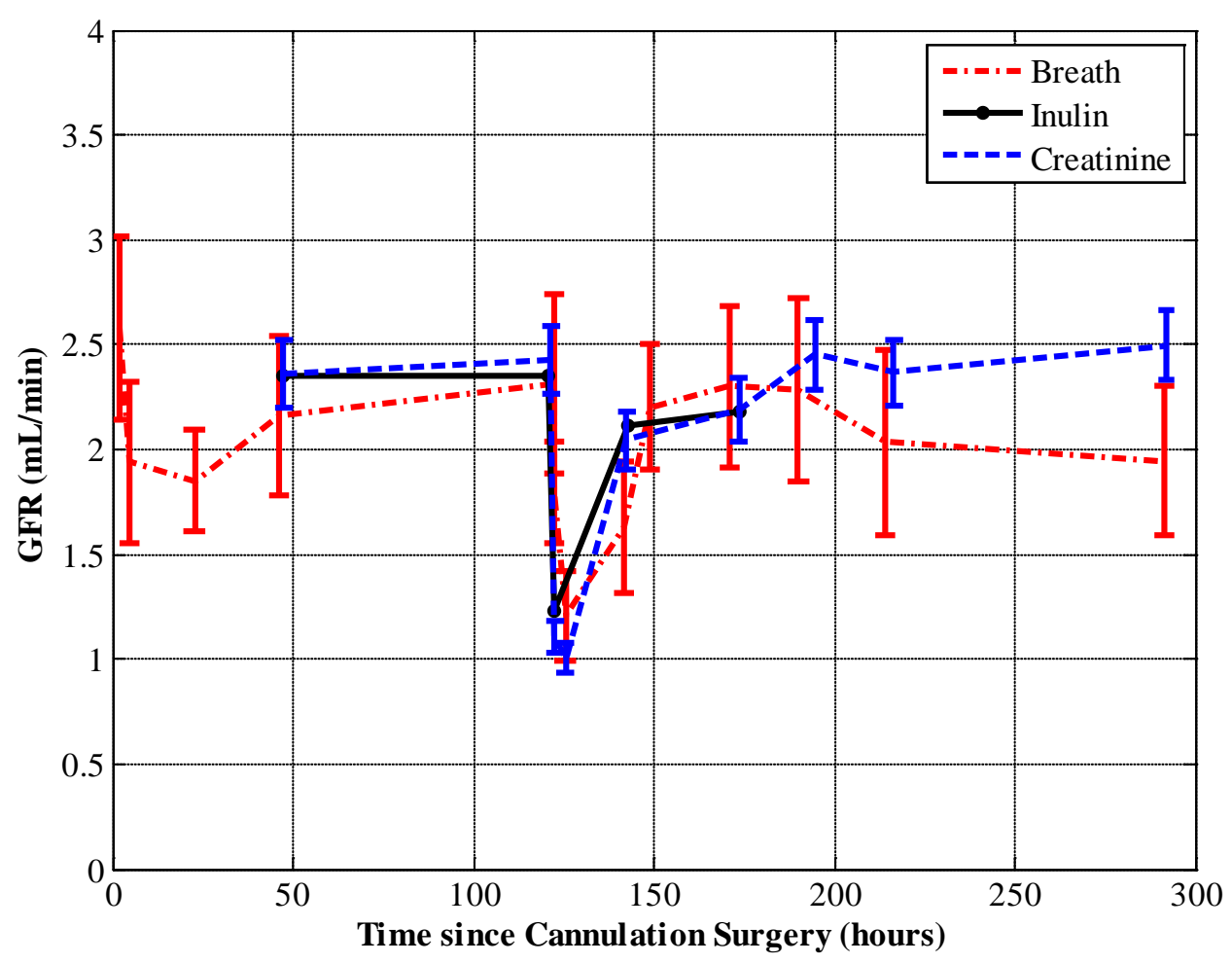

Fig. 15. Rat 6 GFR Comparision 


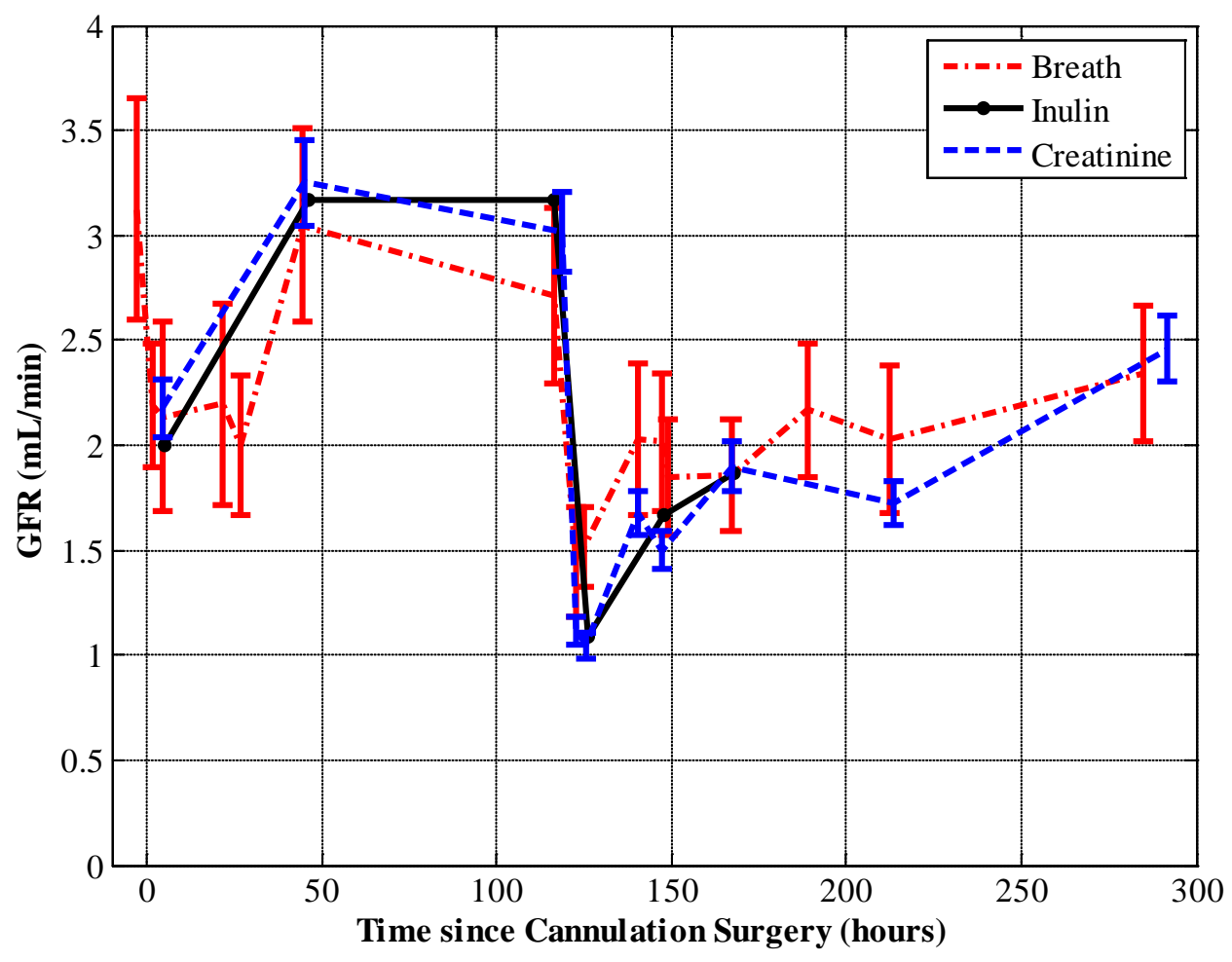

Fig. 16. Rat 7 GFR Comparision

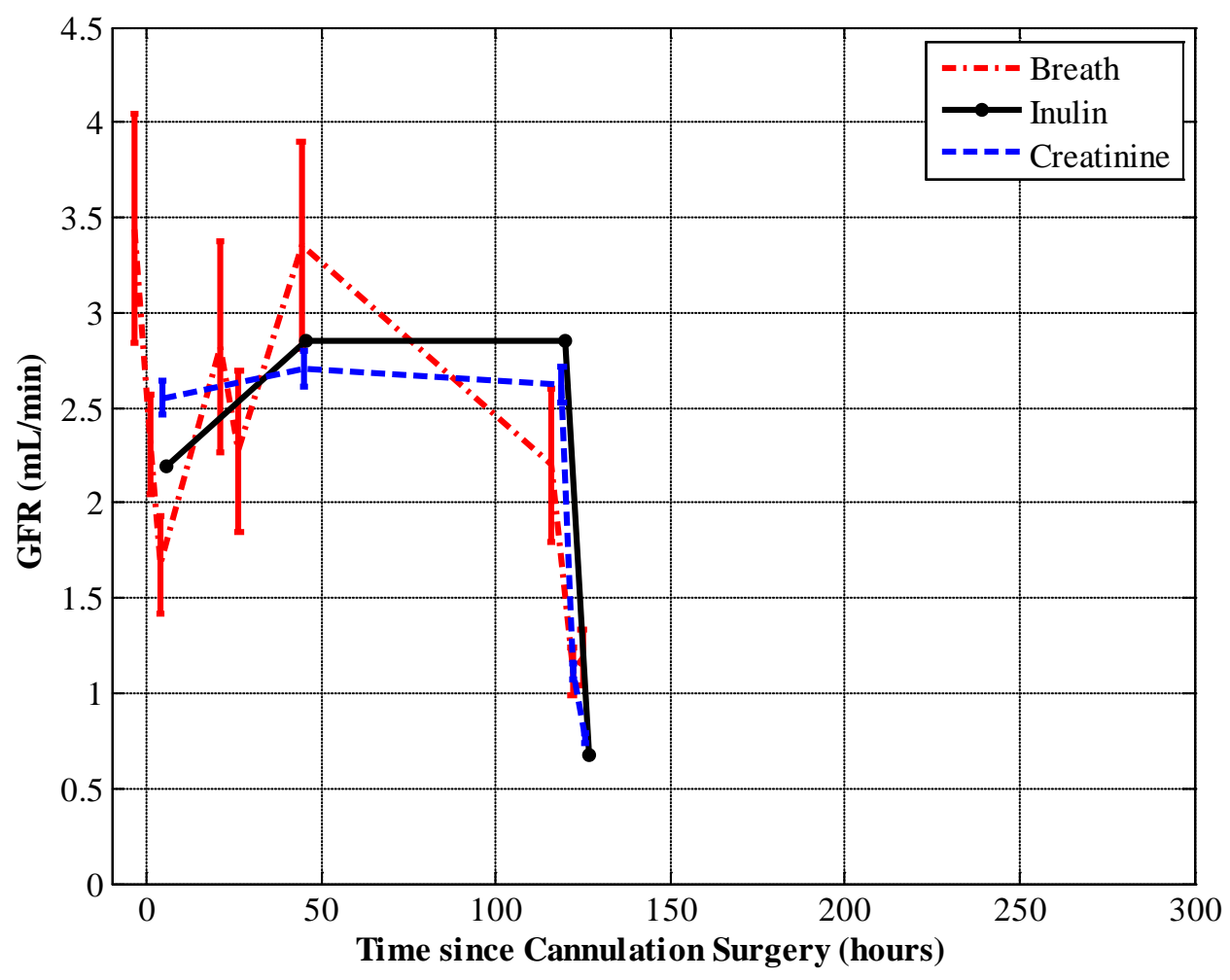

Fig. 17. Rat 8 GFR Comparision 
Figs. 10-17 show the time-course for GFR estimation via the 3 methods for each subject rat. An initial decrease in GFR was caused by surgery alone, and the second, larger decrease in GFR was due to ARF. It is noted that the true GFR would be approximately zero immediately following the renal clamping. However, it would be impractical to perform an inulin clearance test at that time point when urine output is usually zero or minimal for some hours. Using creatinine and breath measures, the GFR estimation would never reach that minimum value due to the time required for the creatinine/ammonia to build up in the blood/breath, and the reality that by the time they did, renal function would already be improving. Error bars indicate the variability of SIFT-MS. Better technology with the Voice $200^{\circledR}$, and filtering of multiple samples would reduce this error and further improve correlations.

\section{Conclusions}

ARF was induced in 8 rats, and the degree of renal failure was estimated using 3 different methods: bolus radio-labelled inulin clearance test, plasma creatinine measurement, and SIFT-MS breath analyte monitoring. The relative decrease in function is the most useful metric, for which the Pearson product-moment correlation coefficient was 0.89 between breath and inulin techniques. However, a population model for absolute GFR estimation was also able to be generated from creatinine and breath data with very good correlations observed in Figs. 10-17.

It was found in a pilot study (Fig. 5), that the concentration of expired ammonia increases as a function of background ammonia concentration with a slope greater than 1. This result indicates that ammonia can be removed from the body at a 
concentration greater than that possible by simple diffusion from the blood, alone, suggesting the presence of ammonia transporters in lung epithelium for actively transporting ammonia.

Determination of renal function via SIFT-MS is attractive since results are available non-invasively and in real time. Correlation between relative decreases in renal function presented here, indicate good promise for fast, non-invasive determination of renal function via breath testing. However, further development would be required to make this a viable and cost-effective clinical solution. 


\section{References}

[1] Chiou, W.L. and Hsu, F.H. (1975). Pharmacokinetics of creatinine in man and its implications in the monitoring of renal function and in dosage regimen modifications in patients with renal insufficiency. Journal of clinical pharmacology.Vol. 15 (5-6) pp. 427-34.

[2] Solomon, R. and Segal, A. (2008). Defining acute kidney injury: what is the most appropriate metric? Nature Clinical Practice: Nephrology, 4(4):208-215.

[3] Schrier, R.W. (2010). Early intervention in acute kidney injury. Nature Reviews Nephrology. Vol. 6 (1) pp. 56-9.

[4] Merriam-Webster. Website, 2006.

[5] Van Acker, B.A.C., Koomen, G.C.M., Arisz, L. (1995). Drawbacks of the constant-infusion technique for measurement of renal function. Am. J. Physiol, 268: F543-F552.

[6] Jones, G.R.D. and Lim, E.M (reviewed) (2003). The National Kidney Foundation Guideline on Estimation of the Glomerular Filtration Rate. Clin Biochem Rev. 2003 August; 24(3): 95-98

[7] Davison (Ed), A.M. et al. (1992). Oxford Textbook of Clinical Nephrology, Chapter 1.3. Oxford University Press, Oxford.

[8] Sturgeon, C., Sam, A.D.II, Law, W.R. (1998). Rapid determination of glomerular filtration rate by a single-bolus inulin: a comparison of estimation analyses. J. Appl. Physiol, 84(6): 2154-2162.

[9] Loon, N., Shemesh, O., Morelli, E., and Myers, B.D. (1989). Effect of angiotensin II infusion on the human glomerular filtration barrier. Am J Physiol. Vol. 257 (4 Pt 2) pp. F608-14. 
[10] Herget-Rosenthal, S., Kribben, A., Pietruck, F., Ross, B., and Philipp, T. (1999). Two by two hour creatinine clearance - repeatable and valid. Clin Nephrol. Vol. 51 (6) pp. 348-354.

[11] Herrera-Gutiérrez, M.E., Seller-Pérez, G., Banderas-Bravo, E., Muñoz-Bono, J., Lebrón-Gallardo, M., and Fernandez-Ortega, J.F. (2007). Replacement of 24-h creatinine clearance by 2-h creatinine clearance in intensive care unit patients: a single-center study. Intensive care medicine. Vol. 33 (11) pp. 1900-6.

[12] Cockcroft, D.W. and Gault, M.H. (1976). Prediction of creatinine clearance from serum creatinine. Nephron, 16(1):31-41.

[13] Smith, D. and Spanel, P. (1996). Application of ion chemistry and the sift technique to the quantitative analysis of trace gases in air and on breath. Int. Rev. Phys. Chem., 15 (1):231271.

[14] Moorhead, K. (2009). Design and Implementation of Analytical mathematics for SIFT-MS Medical Applications. PhD thesis, University of Canterbury.

[15] Schubert, J.K., Miekisch, W., Birken, T., Geiger, K., and Noldge-Schomburg, G.F. (2005) Impact of inspired substance concentrations on the results of breath analysis in mechanically ventilated patients. Biomarkers, 10(2-3):138-152.

[16] Phillips, M. (1997). Method for the collection and assay of volatile organic compounds in breath. Anal Biochem, 247:272-278.

[17] Risby, T.H.and Sehnert, S.S. (1999). Clinical application of breath biomarkers of oxidative stree status. Free Radic Biol Med, 27:1182-1192.

[18] Wyse, C. A., Skeldon, K.D., Cathcart, A.J., Sutherland, R., Ward, S.A., Gibson, G., McMillan, L.C., Padgett, M.J., Preston, T., Yam, P.S., and Love, S. Breath 
(2005). Analysis for Clinical Diagnosis and Therapeutic Monitoring. World Scientific Publishing Co. Pte. Ltd.

[19] Neilson, J. (2006). Integrity of storage media for clinical applications with siftms instruments. Master's thesis, University of Canterbury.

[20] Carson, E. and Cobelli, C. (2001). Modelling methodology for physiology and medicine. Academic Press, San Diego.

[21] Hann, C.E., Chase, J.G., Lin, J., Lotz, T., Doran, C.V., and Shaw, G.M. (2005). Integral-based parameter identification for long-term dynamic verification of a glucose-insulin system model. Computer Methods and Programs in Biomedicine, 77(3): 259-270.

[22] Docherty, P.D., Chase, J.G., Lotz, T., Hann, C.E., Shaw, G.M., Berkeley, J.E., Mann, J.I., and McAuley, K. (2009). DISTq: An Iterative Analysis of Glucose Data for Low-Cost, Real-Time and Accurate Estimation of Insulin Sensitivity, The Open Medical Informatics Journal, 3: pp 65-76.

[23] Levitt, D.G. (2003). The pharmacokinetics of the interstitial space in humans. BMC Clinical Pharmacology, 3: 3.

[24] Poulsen, H.L., Jensen, H.A., and Parving H.H. (1977). Extracellular fluid volume determined by a single injection of inulin in men with untreated essential hypertension. Scand J Clin Lab Invest., 37(8):691-696.

[25] Cooper, A.J.L., and Freed, B.R. (2005). Metabolism of $\left[{ }_{13} \mathrm{~N}\right]$ ammonia in rat lung. Neurochemistry International, 47:103-118.

[26] Pass, D. and G. Freeth. (1993). The rat. ANZCCART News 6(4): 1-4. 